\title{
Bioatividade de óleos essenciais e extratos vegetais no controle de doenças causadas por Phytophthora nicotianae em solanáceas
}

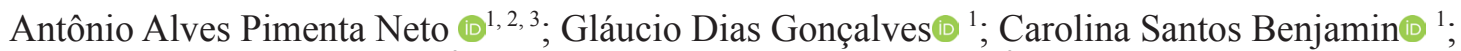 \\ Larissa Corrêa do Bomfim Costa $\mathbb{1}^{2}$; Rosilene Aparecida de Oliveira $\mathbb{C}^{2}$, Sônia Maria Alves de Oliveira $\mathbb{1}^{3}$; \\ Edna Dora Martins Newman Luz (i) ${ }^{1}$
}

${ }^{1}$ Comissão Executiva do Plano da Lavoura Cacaueira, Rodovia Ilhéus-Itabuna km 22, 45660-000, Ilhéus, BA, Brasil; ${ }^{2}$ Universidade Estadual de Santa Cruz, Rodovia Ilhéus-Itabuna km 16, 45662-900, Ilhéus, BA, Brasil; ${ }^{3}$ Universidade Federal Rural de Pernambuco, Rua Dom Manoel de Medeiros, s/n, 2171-900 Recife - PE, Brasil.

Autor para correspondência: Antônio A. Pimenta Neto (pimenta_dm@yahoo.com.br)

Data de chegada: 05/11/2018. Aceito para publicação em: 01/06/2020.

$10.1590 / 0100-5405 / 215960$

\section{RESUMO}

Pimenta Neto, A.A.; Gonçalves, G.D.; Benjamin, C.S.; Costa, L.C.B.; Oliveira, R.A.; Oliveira, S.M.A.; Luz, E.D.M.N. Bioatividade de óleos essenciais e extratos vegetais no controle de doenças causadas por Phytophthora nicotianae em solanáceas. Summa Phytopathologica, v.46, n.3, p.267-272, 2020.

Óleos essenciais (EO) e extratos brutos aquosos (EBA) de Syzygium aromaticum e Cymbopogon nardus, e seus principais componentes químicos foram investigados para controle in vitro e in vivo de doenças causadas por Phytophthora nicotianae em tomateiro (Lycopersicon esculentum) e berinjela (Solanum melongena). Experimentos in vitro foram conduzidos pelo método de diluição em ágar com diferentes concentrações de óleos essenciais (OE's) $(0,1 ; 0,5 ; 1,0 \mu \mathrm{L} / \mathrm{mL})$ e extratos aquosos brutos (EBA's) $(1,0 ; 10,0$ 20,0\%) para avaliar o potencial inibitório sobre o crescimento micelial e germinação de zoósporos. Com as porcentagens de inibição e concentrações inibitórias mínimas encontradas, realizou-se testes em frutos e plântulas sob ambiente controlado. As variáveis avaliadas foram o diâmetro médio das lesões formadas na superfície dos frutos e a incidência e morte de plântulas ao longo de seis e 15 dias de avaliação, respectivamente. Os produtos que mais inibiram o crescimento micelial e a germinação de zoósporos foram obtidos de S. aromaticum, nas concentrações de $0,5 \mu \mathrm{L} / \mathrm{mL}$ e $10 \%$ de OE e EBA, respectivamente. Os tratamentos que mais retardaram a progressão da doença em frutos e plântulas, em comparação com o controle, foram o OE e EBA de C. nardus a $1,0 \mu \mathrm{L} / \mathrm{mL}$ e $20 \%$, respectivamente. Portanto, os produtos obtidos de $S$. aromaticum e C. nardus, têm potencial para reduzir $\mathrm{o}$ ataque deste patógeno em tomate e berinjela.

Palavras-chave: Controle alternativo de doenças de plantas; Phytophthora nicotianae, S. aromaticum, C. nardus.

\section{ABSTRACT}

Pimenta Neto, A.A.; Gonçalves, G.D.; Benjamin, C.S.; Costa, L.C.B.; Oliveira, R.A.; Oliveira, S.M.A.; Luz, E.D.M.N. Bioactivity of essential oils and plant extracts on the control of diseases caused by Phytophthora nicotianae in Solanaceae. Summa Phytopathologica, v.46, n.3, p.267-272, 2020.

Essential oils (OE) and crude aqueous extracts (EBA) from Syzygium aromaticum and Cymbopogon nardus and their major chemical components were investigated for in vitro and in vivo control of diseases caused by Phytophthora nicotianae in tomato (Lycopersicon esculentum) and eggplant (Solanum melongena). In vitro experiments were conducted using the agar dilution method with different concentrations of $\mathrm{OE}(0.1,0.5,1.0 \mu \mathrm{L} / \mathrm{mL})$ and $\operatorname{EBA}(1.0,10.0,20.0 \%)$ to evaluate their inhibitory potential on mycelial growth and zoospore germination. The obtained percent inhibition and minimum inhibitory concentrations were used to performed tests in fruits and seedlings under controlled conditions. The evaluated variables were mean lesion diameter on the surface of fruits and incidence and death of seedlings over six and 15 days of evaluation, respectively. The products that most inhibited mycelial growth and zoospore germination were obtained from $S$. aromaticum, at the concentrations of $0.5 \mu \mathrm{L} / \mathrm{mL}$ and $10 \% \mathrm{OE}$ and EBA, respectively. The treatments that most delayed the disease progress in fruits and seedlings, compared to control, were $\mathrm{OE}$ and EBA of C. naudus at $1.0 \mu \mathrm{L} / \mathrm{mL}$ and $20 \%$, respectively. Thus, products obtained from $S$. aromaticum and $C$. nardus have the potential to reduce the attack by this pathogen in tomato and eggplant.

Keywords: Alternative control of plant diseases, Phytophthora nicotianae, S. aromaticum, C. nardus.

A família Solanaceae reúne várias olerícolas de importância econômica, que são altamente vulneráveis à problemas fitossanitários (9), e devido a isto, é grande a quantidade de agrotóxicos empregada para o controle delas (11).

Embora os agroquímicos sejam largamente utilizados por, apresentarem em curto prazo, um efeito positivo para o produtor, podem induzir o surgimento de isolados dos fitopatógenos resistentes às substâncias químicas utilizadas, a exemplo de isolados de Phytophthora nicotianae Breda de Hann resistentes à metalaxyl (24), e trazer efeitos negativos para a sociedade como um todo e para o meio ambiente devido à poluição causada pelos resíduos. Nesse contexto, a agricultura alternativa ou sustentável estimula a busca de novas medidas de proteção das plantas contra as doenças. Um dos enfoques é o controle alternativo de doenças de plantas através o uso de óleos e extratos vegetais ou de metabólitos secundários $(16,22)$, que oferecem maior segurança, seletividade, biodegradabilidade, viabilidade econômica, e aplicabilidade em programas de manejo integrado de pragas devido ao baixo impacto ambiental.

Várias plantas por possuírem substâncias comprovadamente antimicrobianas, vêm sendo utilizadas no controle alternativo de doenças de plantas, podendo ser aplicados através da atomização na parte área, ou incorporação ao solo, para controlar a densidade populacional de patógenos habitantes do solo como Phytophthora spp. (3). O objetivo deste trabalho foi avaliar a eficiência de óleos e extratos vegetais de Syzygium aromaticum e 
Cybopongon nardus no controle das doenças causadas por P. nicotianae em tomateiro (Lycopersicon esculentum Mill.) e berinjela (Solanum melongena L.).

\section{MATERIAL E MÉTODOS}

\section{Processo de extração de óleos essenciais e extratos vegetais}

Extratos bruto aquosos (EBAs) e óleos essenciais (OEs) foram obtidos a partir de folhas frescas de Cymbopogom nardus 'Cn' (citronela) e capítulos florais de Syzygium aromaticum ' $\mathrm{Sa}$ ' (cravo-da-índia), obtidos no Horto Medicinal da Universidade Estadual de Santa Cruz (UESC), e casas comerciais do Centro de Abastecimento do município de Itabuna-BA, respectivamente.

Folhas e capítulos florais foram desinfestados com hipoclorito de sódio (NaClO) 2\%, água destilada e esterilizada (ADE), na proporção de 100:200, $24 \mathrm{~h}$ antes da sua utilização. Os EBA's foram obtidos através da trituração, filtrados e acondicionados em frascos de vidro âmbar. A solução estoque do extrato de ' $\mathrm{Sa}$ ' foi autoclavada, enquanto que a de ' $\mathrm{Cn}$ ' por possuir compostos antimicrobianos termolábeis, foi suplementada pela adição dos componentes utilizados no meio seletivo PARPH.

O óleo essencial foi extraído por hidrodestilação com arraste de vapor em sistema tipo Clevenger, separado dos hidrolatos por partição gravimétrica, transferido para frascos de vidro âmbar e acondicionados a $5^{\circ} \mathrm{C}$. O rendimento dos OE's foi calculado dividindo a massa obtida pela massa inicial e multiplicando por 100 .

\section{Potencial antifúngico de extratos vegetais e óleos essenciais in vitro}

O crescimento de dois isolados de P. nicotianae (1365 e 1405, da Coleção de Phytophthora Arnaldo Medeiros), selecionados previamente com base na agressividade à frutos de solanáceas, foi testado pelo método bioanalítico frente a incorporação em CA fundente $\left(45-50{ }^{\circ} \mathrm{C}\right)$ das concentrações de 0,$1 ; 0,5$; $1 \mu \mathrm{L} / \mathrm{mL}$ e $1 ; 10$ e $20 \%$ das soluções estoque de OE's, e EBA's . As metalaxyl + mancozeb foi usado nas mesmas concentrações dos OE's. Às placas com OE's foi adicionado o surfactante Tween 80 em igual proporção dos OE's. No centro das placas dos tratamentos e controle (meio + surfactante) depositou-se os isolados, sendo incubadas a $25 \pm 2{ }^{\circ} \mathrm{C}$ por sete dias no escuro. $\mathrm{O}$ experimento foi em delineamento inteiramente casualizados (DIC) com arranjo fatorial $5 \times 3+1$ (produtos e concentrações), com cinco repetições. foram realizadas, Medições ortogonais do diâmetro das colônias foram realizadas diariamente e calculada a porcentagem de inibição por comparação com a testemunha. Nos tratamentos com $100 \%$ de inibição, o potencial fungistático e/ou fungicida foi avaliado. A inibição da germinação dos zoósporos foi avaliada com as mesmas concentrações citadas anteriormente, em meio ágar-água (AA) fundente e o controle foi apenas AA com surfactante. Foi adicionado às placas $300 \mu \mathrm{L}$ de $3 \times 10^{5}$ zoósporos $/ \mathrm{mL}(15,17)$, e estas, incubadas a $25 \pm 2{ }^{\circ} \mathrm{C}$ no escuro por $12 \mathrm{~h}$. $\mathrm{O}$ experimento foi montado em DIC, com fatorial $4 \times 3+1$ (quatro produtos e três concentrações e o controle), e oito repetições/tratamento. Avaliou-se o número de esporos germinados/repetição (microscópio óptico - 400X) calculando a porcentagem de inibição em relação à testemunha.

Potencial antifúngico de compostos químicos presentes em óleos essenciais in vitro

O potencial antifúngico de componentes dos óleos (Sigma-Aldrich $®$ ) de ' $\mathrm{Cn}$ ' (citronelal, citonelol, geraniol) e 'Sa' (eugenol) foi avaliado de forma similar aos óleos essenciais.

Caracterização química quantitativa e qualitativa dos óleos essenciais

A composição química quantitativa foi obtida com cromatógrafo à gás acoplada ao detector de ionização de chama (GC-FID) Varian Saturm 3800, equipado com coluna capilar de sílica fundida VF5-ms (30 m x 0,25 mm) com fase estacionária $5 \%$ fenil-95\% dimetilpolisiloxano $(0,25 \mu \mathrm{m}$ de espessura de filme), tendo hélio 5.0 como gás arraste e fluxo de $1,2 \mathrm{~mL} / \mathrm{min}$ (10 psi); e a quantificação dos componentes por integração eletrônica dos picos detectados no FID por normatização. A análise qualitativa foi realizada em espectrômetro de massas Varian Saturno 2000, usando o método de impacto eletrônico de $70 \mathrm{eV}$. A coluna e as condições de temperaturas foram idênticas as usadas na análise CG-FID. A identificação dos componentes dos óleos foi realizada pela análise dos padrões de fragmentação observado nos espectros de massa, e confirmada por comparação espectros de massas com base de dados fornecidos pelo equipamento (NIST 08), além da comparação dos dos índices de retenção com os compostos conhecidos.

Potencial de controle da podridão algodão em frutos de tomate e berinjela

Frutos verdoengos de tomate e berinjela foram desinfestados ( $\mathrm{NaClO} 2 \%)$, secos à $25 \pm 2{ }^{\circ} \mathrm{C}$, e imersos em solução dos $\mathrm{OE}$ 's de ' $\mathrm{S} a$ ' e ' $\mathrm{Cn}$ ', nas concentrações de 0,5 e $1,0 \mu \mathrm{L} / \mathrm{mL}$, e EBA's de 10 e $20 \%$ respectivamente, além do oxicloreto de cobre na concentração de $100 \mu \mathrm{L} / \mathrm{mL}$ por um período de três minutos. Foi adicionado aos tratamentos Wil fix 2\%. Testemunhas inoculadas e absolutas foram avaliadas. Os frutos foram acondicionados em câmara úmida $\left(25 \pm 2{ }^{\circ} \mathrm{C}\right.$, na ausência de luz), e após 24 horas realizadas inoculações com deposição do isolado 1405 na superfície intacta. Os frutos foram mantidos em câmara úmida até o último dia de avaliação. O experimento foi em blocos casualizados (DBC), com cinco repetições. Aárea lesionada foi mensurada do $3^{\circ}$ ao $6^{\circ} \mathrm{DAI}$ e as Curvas de progresso da doença plotadas, utilizando essas mensurações no tempo. Os dados de severidade em proporção (y), linearizados pela transformação logística $[y=\ln [y /(1-y)]]$ (4), foram ajustados a modelo de regressão linear simples, tendo dias após a inoculação (DAI) como variável independente. Foi calculada a taxa de progresso da doença (TPD) e área abaixo da curva de progresso da doença padronizada (AACPD) (4).

Potencial de controle no "damping off" em plântulas de tomateiro e berinjela

As plântulas foram obtidas por semeadura em mistura de $50 \%$ substrato comercial $\left(\right.$ Plantimax $\left.{ }^{\circledR}\right)+50 \%$ solo esterilizado e transportadas $\left(14^{\circ} \mathrm{dia}\right)$ para câmaras de inoculação (sob condições controladas de umidade relativa, temperatura e alternância luminosa de $12 \mathrm{~h}$-data loger $\mathrm{HOBO} \circledast$ ). Antes da inoculação de $3 \times 10^{5}$ zoósporos $/ \mathrm{mL}$ (15), o substrato em que as plântulas se desenvolviam foi saturado com água. Os OE's e EBA's de ' $\mathrm{Sa}$ ' e ' $\mathrm{Cn}$ ', nas concentrações de 0,5 e 1,0 $\mu \mathrm{L} / \mathrm{mL}$, e EBA's de 10 e $20 \%$ respectivamente, foram pulverizadas no limbo foliar até o ponto de escorrimento, e aplicados ao redor do coleto $48 \mathrm{~h}$ antes da inoculação. Foi utilizado o metalaxyl + mancozeb na concentração de $3 \mathrm{~g} / \mathrm{L}$, além da testemunha inoculada e um controle absoluto. $\mathrm{O}$ experimento foi em DBC, com sete tratamentos, cinco repetições com 10 plântulas cada. Foi identificado o período de incubação (Pi), incidência final (IF), número de plantas mortas (NPM), TPD e AACPD. Os dados de progresso da doença também foram transformados para o modelo logístico.

\section{Análises dos dados}

Os dados obtidos foram analisados quanto à normalidade e submetidos à análise de variância pelo teste $\mathrm{F}$ a $1 \%$ de probabilidade. As médias foram separadas pelo teste de Tukey a $5 \%$. Todas as análises estatísticas foram realizadas utilizando-se a versão 9 do software SAS $^{\circledR}$ (SAS, 1988).

\section{RESULTADOS E DISCUSSÃO}

O rendimento dos óleos de capítulos florais de Syzygium aromaticum ' $\mathrm{Sa}$ ' e folhas de Cymbopogom nardus ' $C n$ ' foi de $12,41 \%(\mathrm{~m} / \mathrm{m})$ e $0,56 \%(\mathrm{~m} / \mathrm{m})$, respectivamente. O óleo de ' $\mathrm{Sa}$ ' apresentou coloração levemente amarelada, e densidade maior do que o hidrolato, já o de 'Cn' foi menos denso e translúcido.

Não houve diferença estatística entre os dois isolados de P. nicotianae quanto à inibição do crescimento micelial e os resultados estão em conjunto (Tabela 1). Os produtos de ' $\mathrm{S} a$ 'e apresentaram efeito fungicida a partir da concentração de $10 \%$ (EBA) e $0,5 \mu \mathrm{L} / \mathrm{mL}$ (OE) inibindo $100 \%$ o crescimento micelial dos dois isolados de P. nicotianae (Tabela 1). Estes dados corroboraram relatos da 
Tabela 1. Percentual da inibição do crescimento micelial (ICM) e germinação de zoósporos (IGZ) de dois isolados de Phytophthora nicotianae (1365, 1405), em relação a testemunhas quando cultivados em meio cenoura-ágar com adição de diferentes concentrações de óleos, extratos vegetais e fungicida (metalaxyl + mancozeb), além de compostos químicos (citronelal, citronelol, eugenol e geraniol) presentes nos óleos essenciais de Cymbopogon nardus (citronela) e Syzygium aromaticum (cravo-da-índia), por sete dias, na ausência de luz, à $25 \pm 2^{\circ} \mathrm{C}$

\begin{tabular}{|c|c|c|c|c|c|c|c|c|c|}
\hline \multirow{2}{*}{ Tratamentos } & \multicolumn{3}{|c|}{ ICM } & \multicolumn{3}{|c|}{ IGZ (1365) } & \multicolumn{3}{|c|}{ IGZ (1405) } \\
\hline & $* \mathrm{C} 1$ & $\mathrm{C2}$ & C3 & $\mathrm{C1}$ & $\mathrm{C} 2$ & $\mathrm{C3}$ & C1 & $\mathrm{C} 2$ & $\mathrm{C3}$ \\
\hline Extrato cravo & $50,96 \mathrm{aB}$ & $100,00 \mathrm{aA}$ & $100,00 \mathrm{aA}$ & $91,37 \mathrm{bB}$ & $100,00 \mathrm{aA}$ & $100,00 \mathrm{aA}$ & $54,88 \mathrm{bB}$ & $100,00 \mathrm{aA}$ & $100,00 \mathrm{aA}$ \\
\hline Óleo cravo & $47,78 \mathrm{aB}$ & $100,00 \mathrm{aA}$ & $100,00 \mathrm{aA}$ & $99,25 \mathrm{aA}$ & $100,00 \mathrm{aA}$ & $100,00 \mathrm{aA}$ & $86,63 \mathrm{aB}$ & $99,62 \mathrm{aA}$ & $100,00 \mathrm{aA}$ \\
\hline Fungicida & $6,91 \mathrm{cC}$ & $29,85 \mathrm{~dB}$ & $41,96 \mathrm{dA}$ & - & - & - & - & - & - \\
\hline $\mathrm{CV}$ & \multicolumn{3}{|c|}{6,64} & \multicolumn{3}{|c|}{2,83} & \multicolumn{3}{|c|}{4,67} \\
\hline Eugenol & $60,28 \mathrm{aB}$ & $100,00 \mathrm{aA}$ & $100,00 \mathrm{aA}$ & $98,57 \mathrm{aA}$ & $100,00 \mathrm{aA}$ & $100,00 \mathrm{aA}$ & $96,84 \mathrm{aA}$ & $100,00 \mathrm{aA}$ & $100,00 \mathrm{aA}$ \\
\hline Geraniol & $0,00 \mathrm{cB}$ & $99,06 \mathrm{aA}$ & $100,00 \mathrm{aA}$ & $65,46 \mathrm{bB}$ & $100,00 \mathrm{aA}$ & $100,00 \mathrm{aA}$ & $75,18 \mathrm{bB}$ & $99,17 \mathrm{aA}$ & $100,00 \mathrm{aA}$ \\
\hline $\mathrm{CV}$ & \multicolumn{3}{|c|}{8,33} & \multicolumn{3}{|c|}{3,34} & \multicolumn{3}{|c|}{4,03} \\
\hline
\end{tabular}

${ }^{*} \mathrm{C} 1-0,1 \mu \mathrm{L} / \mathrm{mL}, \mathrm{C} 2-0,5 \mu \mathrm{L} / \mathrm{mL}, \mathrm{C} 3-1,0 \mu \mathrm{L} / \mathrm{mL}$. Médias seguidas da mesma letra, minúsculas nas colunas e maiúsculas nas linhas não diferem entre si pelo teste Tukey $(\mathrm{P} \leq 0,05)$.

literatura, a exemplo da inibição de 100\% do crescimento micelial de Glomerella cingulata e Colletotrichum gloeosporioides, com apenas a concentração de $10 \%$ de extratos bruto aquosos (EBA) (20), e a capacidade do óleo essencial (OE) em inibir o crescimento in vitro de diversos fungos típicos de pós-colheita (10) e de solo (21).

A maior concentração do $\mathrm{OE}$ de ' $C n$ ' não apresentou efeito fungicida, mas inibiu acima de $90 \%$ o crescimento dos dois isolados e o EBA em sua maior concentração inibiu cerca de $64 \%$ do crescimento micelial dos dois isolados. O percentual máximo de inibição proporcionado pela maior concentração $(1,0$ $\mu \mathrm{L} / \mathrm{mL}$ ) do fungicida foi de $48,1 \%$ para o isolado 1405 , e $40,2 \%$ para o 1365 .

O EBA e OE de 'Sa' tiveram efeito semelhante, causando inibição total da germinação dos zoósporos a partir de $10 \%$ e $0,5 \mu \mathrm{L} / \mathrm{mL}$ (Tabela 1). A concentração de $1 \%$ e $0,1 \mu \mathrm{L} / \mathrm{mL}$ destes produtos, proporcionou um percentual de inibição médio superior a $90 \%$. Apenas o $\mathrm{OE}$ de 'Cn' inibiu $100 \%$ a germinação dos zoósporos dos isolados na maior concentração. Tais resultados indicam a existência de diferenças qualitativas e quantitativas entre óleos e extratos. Wilson et al. (25), não encontraram efeito fungitóxico sobre Botrytis cinerea com o EBA de 'Cn', no entanto, o OE a 6,25\% inibiu a germinação dos esporos, demonstrando que os compostos antifúngicos sintetizados por ' $\mathrm{Cn}$ ' podem concentrar-se no OE e estar presentes em concentrações muito baixas no EBA.

O citronelol, diferente do citronelal, inibiu o crescimento micelial de $P$. nicotianae com a apenas $0,1 \mu \mathrm{L} / \mathrm{mL}$, e a partir de $0,5 \mu \mathrm{L} / \mathrm{mL}$, inibiu acima de $90 \%$. Não houve inibição do crescimento do patógeno com os tratamentos citronelal e geraniol na concentração de $0,1 \mu \mathrm{L} / \mathrm{mL}$. Entretanto, o geraniol apresentou aumento significativo na inibição a partir da concentração de $0,5 \mu \mathrm{L} /$ $\mathrm{mL}$, o que difere do tratamento com citronelal. A partir da concentração de 0,5 $\mu \mathrm{L} / \mathrm{mL}$ não houve diferença estatística entre os tratamentos com os compostos eugenol, citronelol e geraniol (Tabela 1).

O tratamento com eugenol destacou-se dos demais e proporcionou a inibição de $98,57 \%$ dos zoósporos (isolado 1365) na concentração de $0,1 \mu \mathrm{L} / \mathrm{mL}$. Com $0,3 \mu \mathrm{L} / \mathrm{mL}$, o citronelol e geraniol não diferiram estatisticamente do eugenol, apenas do citronelal.

Os terpenos predominaram $(90,9 \%)$ nos dois OEs avaliados (Tabela 2). Estas substâncias orgânicas voláteis e não voláteis, são consideradas metabólitos secundários das plantas, com composição predominantemente mono e sesquiterpenos (13). O composto majoritário identificado no óleo essencial de 'Cn' foi o 3-thujanol (48,02\%), seguido do trans-mirtanol (21,21\%) e citronelol $(12,51 \%)$, todos considerados monoterpenos oxigenados (Tabela 2). Esta classe reúne $60 \%$ das substâncias amostradas, e as demais são consideradas sesquiterpênicos. A variabilidade dos compostos encontrados no $\mathrm{OE}$ de ' $\mathrm{S} a$ ' foi menor do que no de ' $\mathrm{Cn}$ ', entretanto foram identificadas mais compostos pertencentes à classe dos sesquiterpenos $(42,85 \%)$ do que monoterpenos (28,57\%), além da presença do ácido salicílico. O eugenol (84,76\%) foi o composto que apresentou maior concentração no $\mathrm{OE}$ de cravo, seguido do cariofileno $(8,20 \%)$ e acetato de eugenol $(5,51 \%)$. Um composto presente neste $\mathrm{OE}$ não foi identificado. Autores citam citronelal, geraniol e citronelol como compostos de maior concentração no $\mathrm{OE}$ de ' $C n$ ' (1), entretanto a presença e concentração desses compostos varia com a parte da planta utilizada, o estágio de desenvolvimento, condições edafoclimáticas, e de destilação e estocagem $(5,14)$.

Apesar do citronelol ter a concentração de $12,38 \%$ no óleo de citronela avaliado neste experimento, este composto aparece como majoritário em algumas situações (5) e teve ação fungitóxica agora comprovada ao inibir acima de 90 $\%$ tanto o crescimento como a germinação dos zoósporos dos isolados de $P$. nicotianae testados, à partir da concentração de $0,5 \mu \mathrm{L} / \mathrm{mL}$. Os efeitos biológicos dos OEs podem ser um resultado sinérgico de todas as substâncias ou apenas dos componentes majoritários (17). Outros componentes ora identificados no óleo essencial de ' $C n$ ' apresentam características de interesse industrial (8), médico (6) e biotecnológico, com atividade antimicrobiana (7).

As moléculas bioativas encontradas em OEs e EBAs podem agir em concomitância com outras moléculas que já possuem efeito positivo no controle de doenças de plantas ocasionadas por Phytophthora spp., de modo a reduzira concentração/volume de fungicidas utilizados convencionalmente. Os fosfitos (principalmente fosfito de potássio) são compostos que possuem comprovada ação antifúngica contra Phytophthora spp. $(12,19)$, e efeito aditivo quando utilizados em conjunto com fungicidas em menor concentração do que o recomendado (2). Portanto, novos testes poderão ser realizados com a comparação do efeito destas moléculas isoladas e em associação.

O eugenol (fenol monoterpênico) foi o componente majoritário encontrado no óleo essencial de ' $\mathrm{Sa}$ ' avaliado, assim como em trabalhos citados na literatura (18); e por ser o tratamento que mais inibiu o crescimento micelial e germinação de zoósporos do patógeno, este é o provável responsável por sua ação fungitóxica.

Foram selecionadas as concentrações de $0,5 \mu \mathrm{L} / \mathrm{mL}$ do OE e $10 \%$ do EBA de ' $\mathrm{Sa}$ '; e de $1,0 \mu \mathrm{L} / \mathrm{mL}$ do OE e $20 \%$ do EBA de ' $\mathrm{Cn}$ ', para os testes in vivo por serem as mínimas a apresentarem potencial inibitório.

Em frutos de tomate, não houve diferença estatística entre os tratamentos e a testemunha inoculada. Em frutos de berinjela, as lesões evoluíram com mesma taxa (TPD) para todos os tratamentos, sendo identificadas diferenças para $\mathrm{y}_{\operatorname{mix}}$ e AACPD. Os produtos de 'Sa'testados no controle da podridão em frutos de berinjela e tomate, não proporcionaram efeito controlador satisfatório, havendo 
Tabela 2. Composição química de óleos essenciais extraídos de receptáculos florais secos de Syzygium aromaticum (cravo-da-índia) e folhas frescas de Cymbopogon nardus (citronela), obtida por análise de espectrometria de massas com cromatografia gasosa (GC-MS)

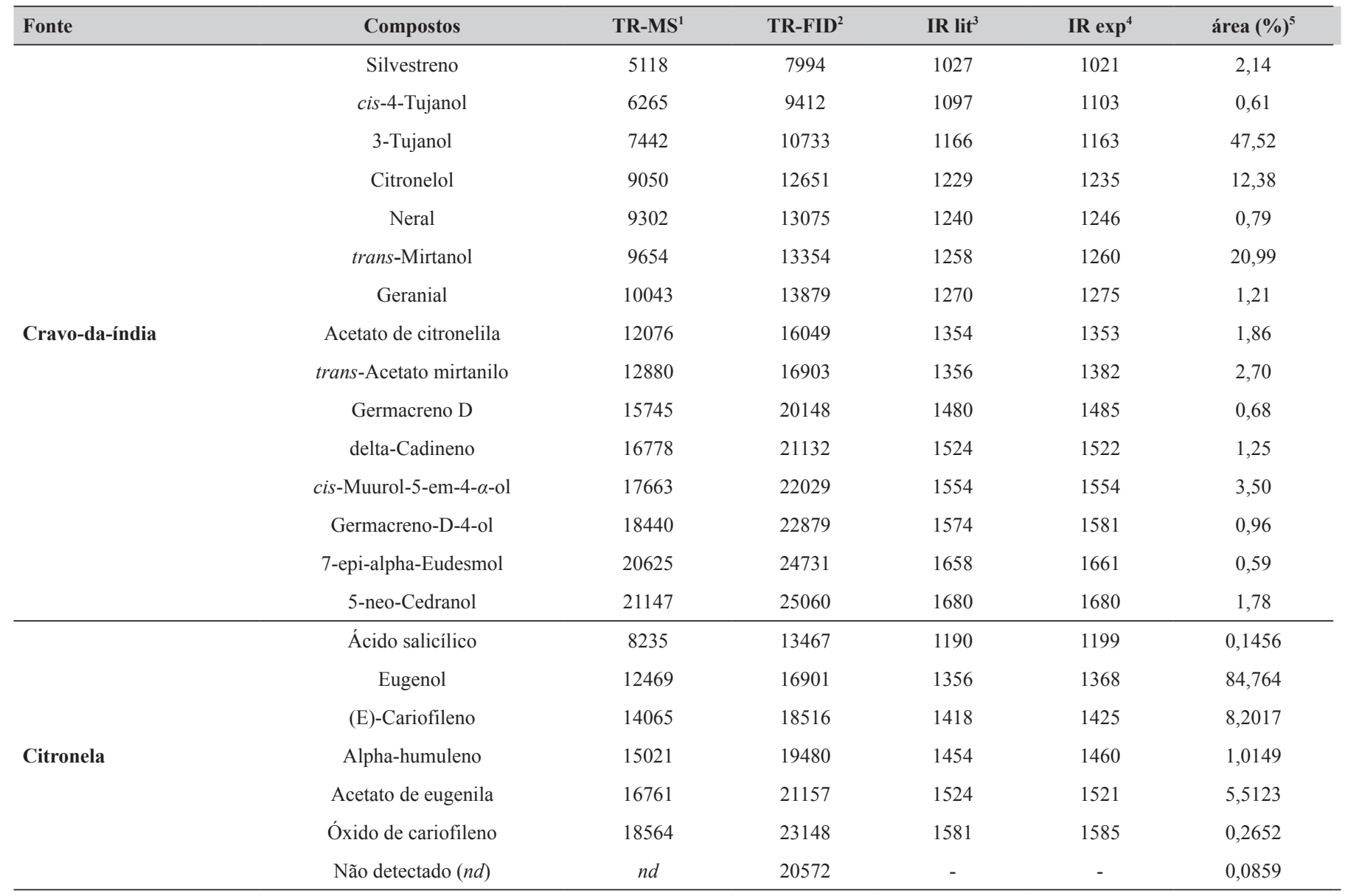

${ }^{1}$ TR-SM tempo de retenção (minutos) em espectrometria de massas; ${ }^{2}$ TR-FID - tempo de retenção em cromatógrafo à gás acoplada ao detector de ionização de chama; ${ }^{3} \mathrm{IR}$ lit - índice de retenção literatura (Adams, 2007), ${ }^{4} \mathrm{IR}$ exp - índice de retenção no experimento; ${ }^{5}$ área (\%) - porcentagem do componente.

apenas pequena redução no tamanho das lesões em frutos de berinjela com EBA (Tabela 3). A $\mathrm{y}_{\text {max }}$ em frutos de berinjela variou de 14,66 (EBA de ' $\mathrm{Sa}$ ') à 22,0 (testemunha inoculada).

O EBA de ' $\mathrm{Sa}$ ' mostrou-se como o mais efetivo dentre os tratamentos alternativos testados em plântulas de tomateiro, por proporcionar a menor $\mathrm{AACPD}$, e não haver diferença entre o período de incubação (PI), incidência final (IF) e número de plantas mortas (NPM) das plântulas tratadas com este produto e com o fungicida que foi $100 \%$ eficiente no controle do patógeno. Os efeitos dos tratamentos com produtos obtidos de ' $\mathrm{Sa}$ ' e o $\mathrm{OE}$ de ' $\mathrm{Cn}$ ' em plântulas de berinjela, não diferiram em relação à testemunha inoculada. $\mathrm{O}$ EBA de ' $C n$ ' foi o único tratamento entre os produtos alternativos testados, que apresentou potencial controlador, apresentando após o fungicida, a menor AACPD, permitindo que $64 \%$ das plântulas de berinjelas inoculadas permanecessem assintomáticas.
A utilização de produtos alternativos (compostos bioativos) no controle de pragas e doenças apresenta vantagens em relação aos produtos sintéticos, já que são facilmente decompostos, não poluem o meio ambiente e não possuem propriedades residuais ou fitotóxicas. Contudo, estes produtos apresentam algumas limitações, como baixa estabilidade e a impossibilidade de monitorar possíveis substâncias tóxicas presentes nos produtos ou resultantes da sua decomposição durante a manipulação (23). Mesmo havendo variações em relação aos tratamentos mais eficazes para o controle, os resultados encontrados além de apresentar produtos e biomoléculas derivadas que atuam de forma positiva no controle de doenças ocasionadas por $P$. nicotianae em solanáceas, enfatizam também a necessidade de testes em condições de cultivo para validação destes produtos alternativos. Ademais, destaca-se a possibilidade de incorporação destas biomoléculas no manejo integrado de doenças causadas por este patógeno, através da combinação de vários compostos e micro-organismos com diversos modos de ação, a fim de aumentar as chances de sucesso do controle. 
Tabela 3. Variáveis epidemiológicas [Severidade inicial (y0), severidade máxima (ymáx), taxa de progresso da doença (TPD), área abaixo da curva de progresso de lesões, período de incubação (PI), incidência final (IF), número de plantas mortas (NPM)] obtidas de frutos e plântulas, de tomate e berinjela, inoculadas com Phytopththora nicotianae, e tratadas com EBA's e OE's de Syzygium aromaticum (cravo-da-índia) Cymbopogon nardus (citronela)

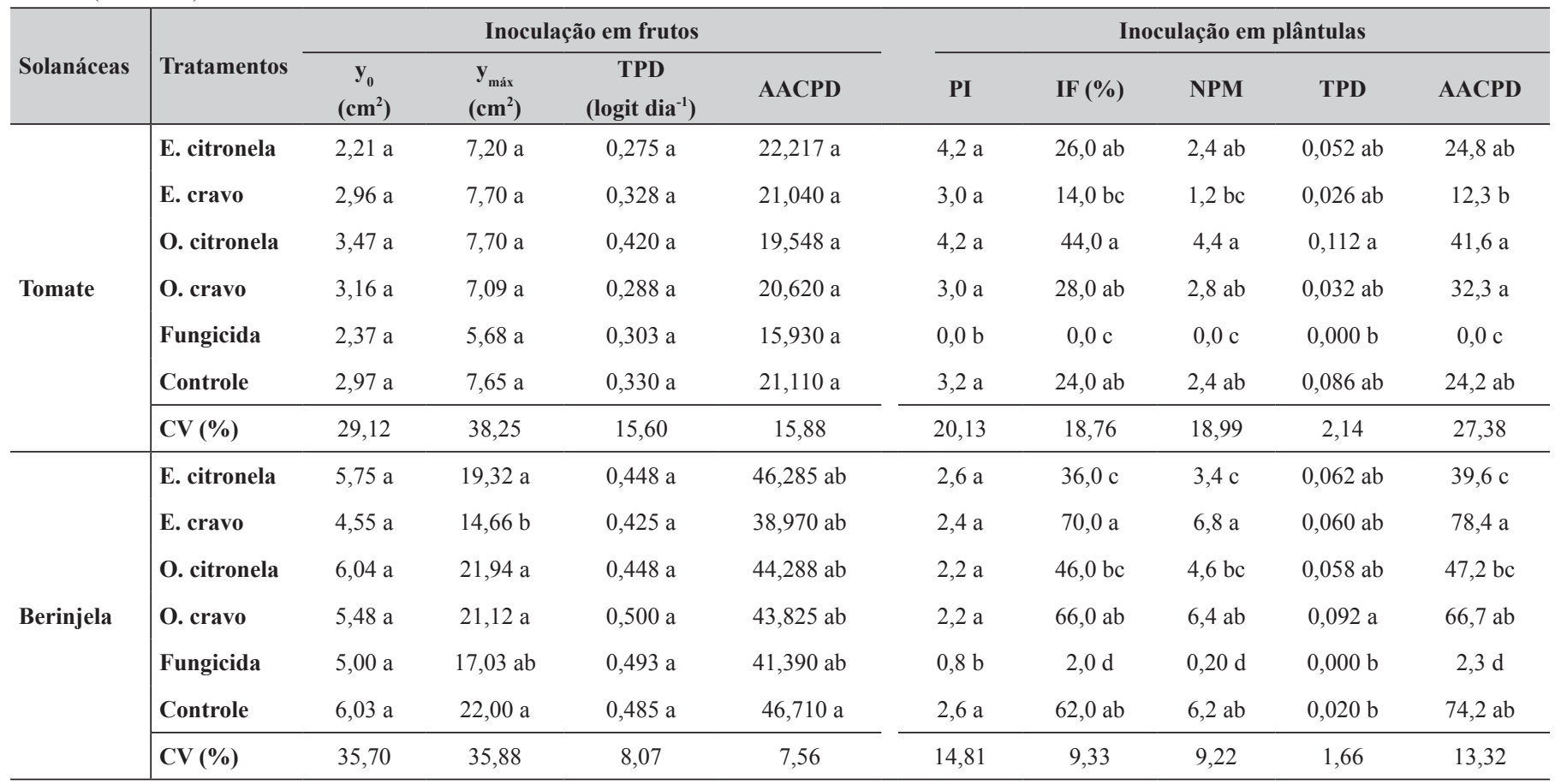

Médias seguidas da mesma letra nas colunas não apresentaram diferenças significativas entre si pelo teste Tukey $(\mathrm{P} \leq 0,05)$.

\section{REFERÊNCIAS}

1. Aguiar, R.W.S.; Ootani, M.A.; Ascencio, S.D.; Ferreira, T.P.S.; dos Santos, M.M.; dos Santos, G.R. Fumigant Antifungal Activity of Corymbia citriodora and Cymbopogon nardus Essential Oils and Citronellal against Three Fungal Species The Scientific World Journal, Cairo, p.1-8, 2014.

2. Borin R.C.; Possenti, J.C.; Rey, M.S.; Bernardi, C.; Mazaro, S.M. Fosfitos associados a fungicidas para controle de doenças e sanidade de sementes de milho. Brazilian Journal of Applied Technology for Agricultural Science, Guarapuava, v.10, n.1, p.83-92, 2017.

3. Bowers, J.H.; Locke, J.C. Effect of formulated plant extracts and oils on population density of Phytophthora nicotianae in soil and control of Phytophthora blight in the greenhouse. Plant Disease, Saint Paul, v.88, p.11-16, 2004.

4. Campbell, C.L.; Madden, L.V. Introduction to plant disease epidemiology. New York: John Wiley, 1990. 532p.

5. Castro, H.G.; Perini, V.B.M.; dos Santos G.R.; Leal, T.C.A.B. Avaliação do teor e composição do óleo essencial de Cybopogon nardus (L.) em diferentes épocas de colheita. Revista Ciência Agronômica, Fortaleza, v.41, n.2, p.308-314, 2010.

6. Charu, M.M. Satija, G., Avinash J.; Nagpal, R.; Kapoor, R.; Ahmad, A. In vitro inhibitory effect of clove essential oil and its two active principles on tooth decalcification by apple juice. International Journal of Dentistry, Cairo, v.2012, 2012.

7. Chiaradia, V.; Paroul, N.; Cansian, R.L.; Júnior, C.V.; Detofol, M.R.; Lerin L.; Oliveira, J.V.; Oliveira, D. Synthesis of eugenol esters by lipase-catalyzed reaction in solvent-free system. Applied Biochemistry and Biotechnology, Clifton, v.168, n.4, p.742-751, 2012.

8. EFSA. Scientific Opinion on the safety and efficacy of allylhydroxybenzenes (chemical group 18) when used as flavourings for all animal species. Euro- pean Food Safety Authority, Parma, v.9, n.12, p.1-14, 2011.

9. Filgueira, F.A.R. Novo Manual de Olericultura: Agrotecnologia moderna na produção e comercialização de hortaliças. Viçosa: Universidade Federal de Viçosa, 2000. 421p.

10. Guynot, M.E. Ramos, A.J.; Setó, L.; Purroy, P.; Sanchis, V.; Marín, S. Antifungal activity of volatile compounds generated by essential oils against fungi commonly causing deterioration of bakery products. Journal of Applied Microbiology, Oxford, v.94, p.893-899, 2003.

11. Kurozawa, C.; Pavan, M.A. Doenças das cucurbitáceas, In: Kimati, H.; Amorim, L.; Rezende, J.A.M.; Bergamin Filho, A.; Camargo, L.E.A. (ed.). Manual de Fitopatologia: Doenças das Plantas Cultivadas. 4.ed. São Paulo: Ceres, 2005. v.2, p.293-302.

12. Töfoli, J.G. Mello, S.C.; Domingues, R.J. Efeito do fosfito de potássio isolado e em mistura com fungicidas no controle da requeima do tomateiro. Arquivos do Instituto Biológico, São Paulo, v.79, n.2, p.201-208, 2012.

13. Maffei, M.E. Sites of synthesis, biochemistry and functional role of plant volatiles. South African Journal of Botany, Pretoria, v.76, n.1, p.612$631,2010$.

14. Oladimeji, F.A. Orafidiya, O.O.; Okeke, I.N.; Dagne, E. Effect of autoxidation on the composition and antimicrobial activity of essential oil of Lippia multiflora. Pharmaceutical and Pharmacological Letters, Stuttgart, v.11, n.2, p.64-67, 2001.

15. Pimenta Neto, A.A. Luz, E.D.M.N.; Gonçalves, G.D.; Oliveira, S.M.A. Influência de meios de cultura, fotoperíodo e ph no crescimento e esporulação de Phytophthora nicotianae. Agrotrópica, Itabuna, v.30, n.3, p.227-236, 2018.

16. Peixinho, G.S.; Ribeiro, V.G.; Amorim, da Rocha, E.P.; Morais, A.C.M. Ação do óleo essencial de Citronela (Cymbopogon nardus L.) sobre o patógeno Lasiodiplodia theobromae em cachos de videira cv. Itália. Summa Phytopathologica, Botucatu, v.45, n.4, p.428-431, 2019.

17. Penuelas, J.; Munné-Bosch, S. Isoprenoids: an evolutionary pool for photo- 
protection. Trends in Plant Science, Oxford, v.10, n.4, p.166-169, 2005.

18. Raina, V.K.; Srivastava, S.K.; Aggarwal, K.K.; Syamasundar, K.V.; Kumar, S. Essential oil composition of Syzigium aromaticum leaf from Little Andaman. Flavour and Fragrance Journal, Chichester, v.16, n.5, p.334-336, 2001.

19. Ramalho, A.C.; Cerioni, L.; Olmedo, G.M.; Volentini, S.I.; Ramallo, J.; Rapisarda, V.A. Control of Phytophthora brown rot of lemons by pre- and postharvest applications of potassium phosphite. European Journal of Plant Pathology, Dordrecht, v.154, n.4, p.975-982, 2019.

20. Rozwalka, L.C.; Lima, M.L.R.Z.C.; Mio, L.L.M.; Nakashima, T. Extratos, decoctos e óleos essenciais de plantas medicinais e aromáticas na inibição de Glomerella cingulata e Colletotrichum gloeosporioides de frutos de goiaba. Ciência Rural, Santa Maria, v.38, n.2, p.301-307, 2008.

21. Santos, L.G.M. Cardoso, M.G.; Lima, R.K.; Souza, P.E.; Guimarães, L.G.L.; Andrade, M.A. Avaliação do potencial fungitóxico do óleo essencial de Syzygium aromaticum (L.) Merr \& Perry (cravo-da-índia). Tecno-Lógica,
Santa Cruz do Sul, v.11, n.1, p.11-14, 2007.

22. Schwan-Esrtrada, K.R.F.; Stangarlin, J.R.; Cruz, M.E.S. Uso de extratos vegetais no controle de fungos fitopatogênicos. Floresta, Curitiba, v.30, n.12, p.129-137, 2000.

23. Silva, M.B.; Rosa, M.B.; Brasileiro, B.G.; Almeida. V.; Silva, C.A. Desenvolvimento de produtos à base de extratos de plantas para o controle de doenças de plantas. In: Venezon, T.J.; Paula Junior, P.A (ed.). Controle alternativo de pragas e doenças. Viçosa: Epamig/CTZM, 2005. p.221-246.

24. Timmer, L.W.; Solel, Z.; Gottwald, T.R.; Ibañez, A.M.; Zitko, S.E. Environmental Factors Affecting Production, release, and Field populations of conidia of Alternaria alternata, the cause of brown spot of Citrus. Phytopathology, Saint Paul, v.88, n.11, p.1218-1223, 1998.

25. Wilson, C.L.; Solar, J.M.; El Ghaouth, A.; Wisniewski, M.E. Rapid evaluation of plant extracts and essential oils for antifungal activity against Botrytis cinerea. Plant Disease, Saint Paul, v.81, n.2, p.204-210, 1997. 